International Journal of Pure and Applied Mathematics

Volume 106 No. 3 2016, 769-789

ISSN: 1311-8080 (printed version); ISSN: 1314-3395 (on-line version)

url: http://www.ijpam.eu

doi: 10.12732/ijpam.v106i3.7

ijpam.eu

\title{
IMPLICIT TRIGGER PRICE DETERMINATION FOR CONTINGENT CONVERTIBLE BOND
}

\author{
Luca Di Persio ${ }^{1}$, Michele Bonollo ${ }^{2}$, Luca Prezioso ${ }^{3}$ \\ ${ }^{1,3}$ Department of Computer Science \\ University of Verona \\ Strada le Grazie, 15-37134 Verona, ITALY \\ ${ }^{2}$ Iason - IMT Lucca \\ Via XX Settembre, 9-37100 Verona, ITALY
}

\begin{abstract}
In this paper we provide concrete evaluations for the trigger price that causes the conversion of Convertible Contingent ( $\mathrm{CoCo})$ bond contracts.

In particular we exploit prices for CoCo bonds traded in real financial markets and the values obtained by the credit derivative as well as by the equity derivative method, to determine the associated implicit trigger price. Because of the computational characteristics of the proposed approaches, we also provide related algorithms.
\end{abstract}

AMS Subject Classification: 60H15, 60H35, 91B60, 91G20, 91G60

Key Words: convertible contingent bonds, credit derivative approach, equity derivative approach, mathematical finance, stochastic differential equations

\section{Introduction}

As a main consequence of the recent financial crisis, governments developed new regulatory frameworks on adequacy of banks' capital. Therefore financial institutions began to develop tools to strengthen the capital, as in the case of the so called convertible bond contracts, or CoCo bonds for short. In this paper we consider different methodologies to evaluate CoCo contracts, focusing in particular on the Equity Derivative one. We would like to recall that CoCo

Received: January 30, 2016

Published: February 26, 2016

${ }^{\S}$ Correspondence author (c) 2016 Academic Publications, Ltd.

url: www.acadpubl.eu 
contracts are hybrid bonds that are convertible into shares, provided certain conditions are satisfied. The latter are the so called trigger events, namely the investor will no longer have the right to receive coupons at fixed dates, but will cash dividends if the company and the board of directors make profits and then decide to distribute a part of them as, e.g., a part of the bank's capital that has issued them. Therefore this conversion will substantially lighten the debt exposure. In particular, a conversion trigger event is determined by the fact that the core tier ratio 1, see Def. 3 below, takes values under a predetermined threshold. In this case the risk for the investor will be higher, due to possible significant losses. The main characteristics of the CoCo bonds will be analyzed in what follows, starting by defining bonds and hybrid bonds, for more details see, e.g., [7], [5], [4], [9], [2] and references therein, while we refer to, e.g., [1], [10], for an alternative methods to evaluate CoCo bonds, namely those based on structural models. The first part of the article, i.e. Sec. (2), will be devoted to the definitions of some financial technicalities, see, e.g., [8] and [6], for a more extensive presentation. In Sec. (3) we provide a detailed description of both the Credit Derivative and the Equity approach. Moreover, contracts with different technical as well as financial characteristics will be taken into account, see Sec. (4), by considering the parameters of CoCo bond contracts which are currently present in real financial markets. and Related algorithms, written by making use of pseudocode, are presented in Sec. (5). Eventually, in Sec. (6), we provide a glossary of main financial terms used throughout all the paper as to give a self contained treatment of the subject.

\section{The Financial Framework}

In what follows, we recall the main characteristics of some basic financial tools that are linked to the CoCo bond definition

Definition 1 (Bond). A bond is a credit instrument that is part of the debt taken out by a corporation or by a public institution for its own funding. Such type of loan implies that the issuer itself is configured as debtor and the investor as a creditor. In face of the initial capital, the investor may receive periodic interest, the coupons, in addition to the repayment of the loan at maturity. It is worth to mention that a bond always returns the value, also in the case of early repayment.

Definition 2 (Hybrid bonds). Hybrid bonds are tools with intermediate features, between traditional bonds and shares. Then such type of contracts is ranked in an intermediate position between debt and equity. Accordingly, they 
are more profitable for the investor, but riskier than the pure debt. The main improvement brought to the issuer is to not worsen the relationship between debt and equity as in the case of normal bonds, in fact hybrid bonds are not considered as pure debt, but they participate to risk capital.

It is useful to recall also some of the principal features of the CoCo bonds, namely

- Subordination: the share has a seniority which is greater only with respect to the equities one. Moreover it is subordinate to all the other Company emissions;

- Expirtion: usually in the long term;

- Call clause: the issuer has the possibility to repay the loan early.

Summing up previous CoCo features, it follows that such type of contracts are essentially bonds issued by banks, with the possibility to be converted in shares, if the emitter requires capital or if a trigger event happens. In particular, the bank issuing such bonds has to announce the investors that, if certain capital ratios, e.g. the tier 1 , fall below a preset value, the debt could be converted into shares; or the bank could automatically turn the debt into capital in the form of shares, to the immediate benefit of its own funds. As mentioned before, a particular relevant parameter, widely used in the banking sector, is the so called core tier 1 , namely

Definition 3 (Core Tier 1 ratio). The core tier 1 ratio is the ratio of primary capital, namely the paid-up capital covering profits and eliminating losses, and the assets weighted by their level of risk:

$$
\text { Core Tier } 1 \text { Capital Ratio }=\frac{\text { Core Tier } 1 \text { Capital }}{R W A} \text {, }
$$

where we have used the parameter $R W A$ indicates the risk-weighted assets.

It is worth to mention that the fair price and the dynamics of the CoCo are mainly determined by the type of conversion, the face value fraction that will be converted into shares, the trigger event and the conversion price. In the following section we briefly describe each of these components. Let us recall that a trigger event, with respect to bonds emission, is defined as a certain financial condition that causes the conversion of the bond into shares, or determines a scenario of devaluation, see, e.g., [5]. Most used trigger scenarios are the following: 
- Acoounting Trigger (AT): A patrimonial indicator is chosen to represent the financial ability of the bank, e.g., the core tier 1 ratio. Since it is defined as the ratio of the bank's core equity capital and its total riskweighted assets, the significance of an AT, as the core tier 1, depends on whether or not the issuer adopts the internal models. In addition, such indices are not available all the time, but usually are published quarterly. See the Glossary for more details

- Market Trigger (MT): The MT is defined using forward looking parameters such as, e.g., Credit Default Spreads (CDS), or share price. When the stock price exceeds a predefined barrier, then such an event causes the conversion. This type of trigger is subject to market manipulations. In fact the latter has been studied almost exclusively in the academic field, while, in real markets, it is likely to take in to consideration some variants often related to accounting indexes.

- Regulatory Trigger (RT): Such a trigger is related to governments actions. In particular it is possible that, under particular economical and/or political conditions, a certain government decides when and how to proceed with a write-down conversion type.

- Multivariate Trigger (MultiT): More triggers are combined together as to define a multivariate trigger. As an example, MT or AT may be calibrated by an RT if the government declare a state of emergency for the bank, and if the index of solvability exceeds a predetermined threshold which causes the bond to be converted into shares.

Concerning concrete methods of conversion, they can be of different types, nevertheless they can be analyzed with respect to, e.g., CoCo bond characterized by an MT type trigger, without loss of generality. In the aforementioned case, if the trigger event occurs, the contract could be converted into a predetermined number of shares, or the value of the debt may be written down.

Definition 4 (Conversion Price). The conversion price, $C_{P}$, is the amount of converted face value, $\alpha N$, divided by the conversion ratio, $C_{r}$, namely

$$
C_{p}=\frac{\alpha N}{C_{r}},
$$

where $\alpha \in(0,1]$ is the conversion fraction, $N$ represents the face value and $C_{r}$ is the number of shares received for each converted bond. 
Therefore for the investor will be better a lower conversion price, since this will result in a greater number of shares when the CoCo bond will be converted, while it will be more disadvantageous for the current shareholders, because of higher dilution. Within real markets, the most used conversion prices are

- $C_{p}=S^{*}$ : it equals the share price observed at the trigger time $T^{*}$; which implies a low conversion price, and therefore a high conversion fraction;

- $C_{p}=S_{0}$ : it equals the CoCo price at the issuing moment, hence it implies a low fraction of conversion without producing a high dilution;

- $C_{p}=\max \left(S^{*}, S_{F}\right)$ (with floor): the price is set equal to the price at the conversion time, but with the condition that it is not below the floor $S_{F}$

\section{Evaluation: Credit and Equity Approaches}

In this section we focus on two of the most relevant evaluation methods which are used treating CoCo bonds, namely the Credit Derivative approach, characterized by the determination of the extra yield required to accept the risk of conversion, and the Equity Derivative approach which is considered when the CoCo bond is viewed as a long position of $C_{r}$ shares in the case that the trigger event occurs, see, e.g., [5] [4].

\subsection{Credit Derivative Approach}

Let $r$ be the continuous risk-free interest rate, $q$ be the continuous dividend yield and $\sigma$ be the volatility of the of the CoCo bond underlying. Suppose that the value of the underlying has a dynamic defined by a geometric Brownian motion, namely

$$
d S_{t}=(r-q) S_{t} d t+\sigma S_{t} d W_{t},
$$

where $W_{t}$ is a Brownian motion with respect to the risk-neutral probability measure. Moreover let us define the trigger price $S^{*}$, while we will indicate by $\tau$ the time at which the trigger event occurs. Hence we are interested in determining the trigger event occurrence probability $p^{*}$ defined as $p^{*}:=\mathrm{P}(\tau \in$ $(0, T])$, where $\tau:=\inf \left\{t \geq 0: S_{t} \leq S^{*}\right\}$, see, e.g., [9], for more details. Thanks to the Itô-D oblin formula, we get that the price of the underlying reads as follow

$$
d \ln \left(\frac{S_{t}}{S_{0}}\right)=\left(r-q-\sigma^{2} / 2\right) d t+\sigma d W_{t},
$$


moreover the probability that such a price does not exceed $S^{*}$ at maturity time $T$, equals

$$
\begin{aligned}
\mathrm{P}\left(S_{T} \leq S^{*}\right) & =\mathrm{P}\left(\ln \left(S_{T} / S_{0} \leq \ln \left(S^{*} / S_{0}\right)\right)\right) \\
& =\mathrm{P}\left(\left(r-q-\sigma^{2} / 2\right) T+\sigma W_{T} \leq \ln \left(S^{*} / S_{0}\right)\right) \\
& =\mathrm{P}\left(W_{T} \leq \frac{\ln \left(S^{*} / S_{0}\right)-\left(r-q-\sigma^{2} / 2\right) T}{\sigma}\right),
\end{aligned}
$$

and since $W_{T} \sim \mathrm{N}(0, T)$, we have

$$
\mathrm{P}\left(S_{T} \leq S^{*}\right)=\Phi\left(\frac{\ln \left(S^{*} / S_{0}\right)-\left(r-q-\sigma^{2} / 2\right) T}{\sigma \sqrt{T}}\right) .
$$

If we consider the trigger event occurrence probability, we have

$$
\begin{aligned}
\mathrm{P}(\tau \leq T) & =\mathrm{P}\left(\tau \leq T \cap S_{T} \geq S^{*}\right)+P\left(\tau \leq T \cap S_{T} \leq S^{*}\right) \\
& =\mathrm{P}\left(\tau \leq T \cap S_{T} \geq S^{*}\right)+P\left(S_{T} \leq S^{*}\right)
\end{aligned}
$$

where the first member can be rewritten as the following expected value

$$
\mathrm{P}\left(\tau \leq T \cap S_{T} \geq S^{*}\right)=\mathrm{E}\left[\mathbb{1}_{\{\tau \leq T\}} \mathbb{1}_{\left\{S_{T} \geq S^{*}\right\}}\right]
$$

By defining $\hat{W}_{t}=W_{t}+\theta t$, with $\theta=\left(r-q-\sigma^{2} / 2\right) / \sigma$, and applying the Girsanov theorem, we have the existence of a new measure $Q$ such that $\mathrm{P}^{Q}(A)=$ $\mathrm{E}\left[\mathbb{1}_{A} e^{-\theta W_{t}-\frac{1}{2} \theta^{2} t}\right]$, moreover, under $Q$ the dynamic of the underlying $S$ can be rewritten as follows $d S_{t}=\sigma S_{t} d \hat{W}_{t}$, implying $d\left(\ln S_{t}\right)=\sigma d \hat{W}_{t}$, so that the first member of eq. (1) becomes

$$
\begin{aligned}
& \mathrm{P}\left(\tau \leq T \cap S_{T} \geq S^{*}\right)=\mathrm{E}\left[\mathbb{1}_{\{\tau \leq T\}} \mathbb{1}_{\left\{S_{T} \geq S^{*}\right\}} e^{-\theta W_{T}-\frac{1}{2} \theta^{2} T} e^{\theta W_{T}+\frac{1}{2} \theta^{2} T}\right] \\
& =\mathrm{E}^{Q}\left[\mathbb{1}_{\{\tau \leq T\}} \mathbb{1}_{\left\{S_{T} \geq S^{*}\right\}} e^{\theta \hat{W}_{T}-\frac{1}{2} \theta^{2} T}\right] \\
& =\mathrm{E}^{Q}\left[\mathbb{1}_{\{\tau \leq T\}} \mathbb{1}_{\left\{\hat{W}_{T} \geq \frac{\ln \left(S^{*} / S_{0}\right)}{\sigma}\right\}} e^{\left.\theta \hat{W}_{T}-\frac{1}{2} \theta^{2} T\right]}\right. \\
& =\mathrm{E}^{Q}\left[\mathbb{1}_{\left\{\hat{W}_{T} \leq \frac{\ln \left(S^{*} / S_{0}\right)}{\sigma}\right\}} \exp \left(\theta\left(2 \frac{\ln \left(S^{*} / S_{0}\right)}{\sigma}-\hat{W}_{T}\right)-\frac{1}{2} \theta^{2} T\right)\right] \\
& =\frac{\exp \left(2 \theta \frac{\ln \left(S^{*} / S_{0}\right)}{\sigma}\right)}{\sqrt{2 \pi}} \int_{-\infty}^{\frac{\ln \left(S^{*} / S_{0}\right)}{\sigma}} e^{-\theta \hat{W}_{T}-\frac{1}{2} \theta^{2} T} e^{-\frac{\hat{W}_{T}^{2}}{2 T}} d \hat{W}_{T} \\
& =\frac{\exp \left(2 \theta \frac{\ln \left(S^{*} / S_{0}\right)}{\sigma}\right)}{\sqrt{2 \pi}} \int_{-\infty}^{\frac{\ln \left(S^{*} / S_{0}\right)}{\sigma}} e^{-\frac{\left(\hat{W}_{T}+\theta T\right)^{2}}{2 T}} d \hat{W}_{T}
\end{aligned}
$$




$$
=\left(\frac{S^{*}}{S_{0}}\right)^{2 \frac{r-q-\sigma^{2} / 2}{\sigma^{2}}} \Phi\left(\frac{\ln \left(S^{*} / S_{0}\right)+\left(r-q-\sigma^{2} / 2\right) T}{\sigma \sqrt{T}}\right) .
$$

where we have used the reflection principle for Wiener processes in strong form. Hence

$$
\frac{\mathrm{P}(\tau \leq T)}{\mathrm{P}\left(S_{T} \leq S^{*}\right)}=1+\frac{\mathrm{P}\left(\tau \leq T \cap S_{T} \geq S^{*}\right)}{\mathrm{P}\left(S_{T} \leq S^{*}\right)},
$$

which implies that the probability that the trigger event occurs is

$$
\begin{array}{r}
p^{*}=\Phi\left(d_{1}\right)+\left(\frac{S^{*}}{S_{0}}\right)^{\frac{2 \mu}{\sigma^{2}}} \Phi\left(d_{2}\right), \\
d_{1}:=\frac{\log \frac{S^{*}}{S_{0}}-\mu T}{\sigma \sqrt{T}} ; d_{2}:=d_{1}+\frac{2 \mu \sqrt{T}}{\sigma},
\end{array}
$$

where $\mu:=r-q-\frac{\sigma^{2}}{2}$ and $\Phi$ is the cumulative distribution function of a standard normal random variable. The occurrence probability of the first trigger event in the time interval $[t, t+d t]$ equals $\lambda_{\text {Trigger }} d t, \lambda_{\text {Trigger }}$ being defined as the trigger intensity as a function of $p^{*}$ : In particular $\forall t \in[0, T]$ and taking into account the limit $d t \rightarrow 0$, we have

$$
\mathrm{P}(\tau \leq t+d t \mid \tau \geq t)=\lambda_{\text {Trigger }} d t+o(d t)
$$

which implies

$$
\mathrm{P}(\tau \geq t+s \mid \tau \geq t)=e^{-\lambda_{\text {Trigger }} s},
$$

therefore, choosing $t=0$ and $s=T$, we have

$$
\mathrm{P}(\tau \leq T \mid \tau \geq 0)=1-e^{-\lambda_{\text {Trigger }} T},
$$

so that

$$
\lambda_{\text {Trigger }}=-\frac{\log \left(1-p^{*}\right)}{T} .
$$

Further insights on the CoCo bond model we are considering, come when considering the so called recovery rate, which represents the ratio between the share price at the conversion moment, and the conversion price, namely

$$
R_{C o C o}:=\frac{S^{*}}{C_{p}}
$$

Under risk-neutral assumptions, the spread equalized the expected loss, and since the expected loss is the product of the percentage loss by the probability 
that the trigger takes place, then we get that the $\mathrm{CoCO}$ credit spread, let us indicate it by $\mathrm{cs}_{\mathrm{CoCo}}$, on contingent debt, namley on potential liabilities, equals

$$
c s_{C o C o}=\left(1-R_{C o C o}\right) \lambda_{\text {Trigger }},
$$

therefore the total continuous yield is determined by the sum of the credit spread and risk-free interest rate.

The algorithm 1 presented in Sec. 5, provides a concrete computational example of the Credit Derivative approach.

\subsection{Equity Derivative Approach}

In what follows we consider the Equity Derivative Approach (ED) for the CoCo bonds, taking into account the fact that coupons are no longer paid once the trigger event occurred, a characteristic which is not considered in the Credit Derivative approach. Let us consider a conversion price, that is equal to the price at the trigger moment, but greater that a certain threshold. In this case the conversion price would be equal to the share price at trigger time $T^{*}$, provided that the trigger prices $S^{*} \geq S_{F}$, then $R_{C o C o}=1$ and, according to the Credit Derivative approach, the credit default would be $C S_{C o C o}=0$. However latter scenario cannot be realized, because the coupons flow is interrupted by the conversion. A major benefit of the (ED) consists in overcome latter such an issue. Let us start considering a zero-coupon $\mathrm{CoCo}$ ( $\mathrm{ZC}-\mathrm{CoCo})$ bond. In this case the CoCo can be equivalently defined as follows

$$
\text { ZC-CoCo }=\text { Zero Coupon Corporate Bond + Knock-In Forward, }
$$

where Knock-In Forward equals the long position on Knock-In Call plus the short position on Knock-In Put Forward. It follows that the value of the ZC$\mathrm{CoCo}$ at the initial time is given by

$$
P_{0}=N e^{-r T}+\text { Knock-In Forward . }
$$

In the case with coupons, the CoCo Bond can be seen as a $\mathrm{ZC}$-CoCo adding short positions on binary down-and-in options with expiration dates that coincide with the times at which the coupons would be paid, that is

$$
-\sum_{i=1}^{k} c_{i} e^{-r t_{i}} \mathbb{1}_{\left\{\tau \leq t_{i}\right\}},
$$

where $k$ is the number of unpaid coupons. 
Therefore the CoCo bond price equals the value of a corporate bond (A), plus the value of a knock-in forward (B) and the value of the short positions on the down-in binary options, the latter being written on the value of the coupons at the occurrence of trigger event (C). Summing up, we have $P=A+B+C$, where:

$$
\begin{aligned}
A:= & N e^{-r T}+\sum_{i=1}^{k} c_{i} e^{-r t_{i}} \\
B:= & C_{r}\left(S_{0} e^{-q T}\left(\frac{S^{*}}{S_{0}}\right)^{2 \lambda} \Phi\left(d_{1}\right)-K e^{-r T}\left(\frac{S^{*}}{S_{0}}\right)^{2 \lambda-2} \Phi\left(d_{1}-\sigma \sqrt{T}\right)\right. \\
& \left.-K e^{-r T} \Phi\left(d_{2}+\sigma \sqrt{T}\right)+S e^{-q T} \Phi\left(d_{2}\right)\right) \\
C:= & \sum_{i=1}^{k} c_{i} e^{-r t_{i}}\left(\left(\frac{S^{*}}{S_{0}}\right)^{2 \lambda-2} \Phi\left(d_{1} i-\sigma \sqrt{t_{i}}\right)+\Phi\left(d_{2} i+\sigma \sqrt{t_{i}}\right)\right),
\end{aligned}
$$

with

$$
\begin{aligned}
& K:=C_{p}, C_{r}:=N / C_{p}, d_{1}:=\frac{\log \left(S^{*} / S\right)}{\sigma \sqrt{T}}+\lambda \sigma \sqrt{T} \\
& d_{2}=d_{1}-2 \lambda \sigma \sqrt{T}, d_{1 i}=\frac{\log \left(S^{*} / S\right)}{\sigma \sqrt{t_{i}}}+\lambda \sigma \sqrt{t_{i}} \\
& d_{2 i}=d_{1}-2 \lambda \sigma \sqrt{t_{i}}, \lambda=\frac{r-q+\sigma^{2} / 2}{\sigma^{2}} .
\end{aligned}
$$

In Sec. (5), see algorithm 2, we provide a numerical example of the previous derivations. The algorithm is developed on the basis of the flowchart presented in Fig.(1).

\section{Case Studies}

Traded CoCo contracts do not explicit the trigger price of the underlying, instead usually they specify a Core Tier 1 trigger. The latter choice implies possible estimation problems mainly due to the fact that Core Tier 1 ratio is not continuously available, unlike what happens for the underlying price, but only periodically. It follows that between two consecutive, e.g. quarterly, financial reports, the Core Tier 1 can be only estimated. In what follows we consider a variation of the setting analyzed in the previous section, by considering an 


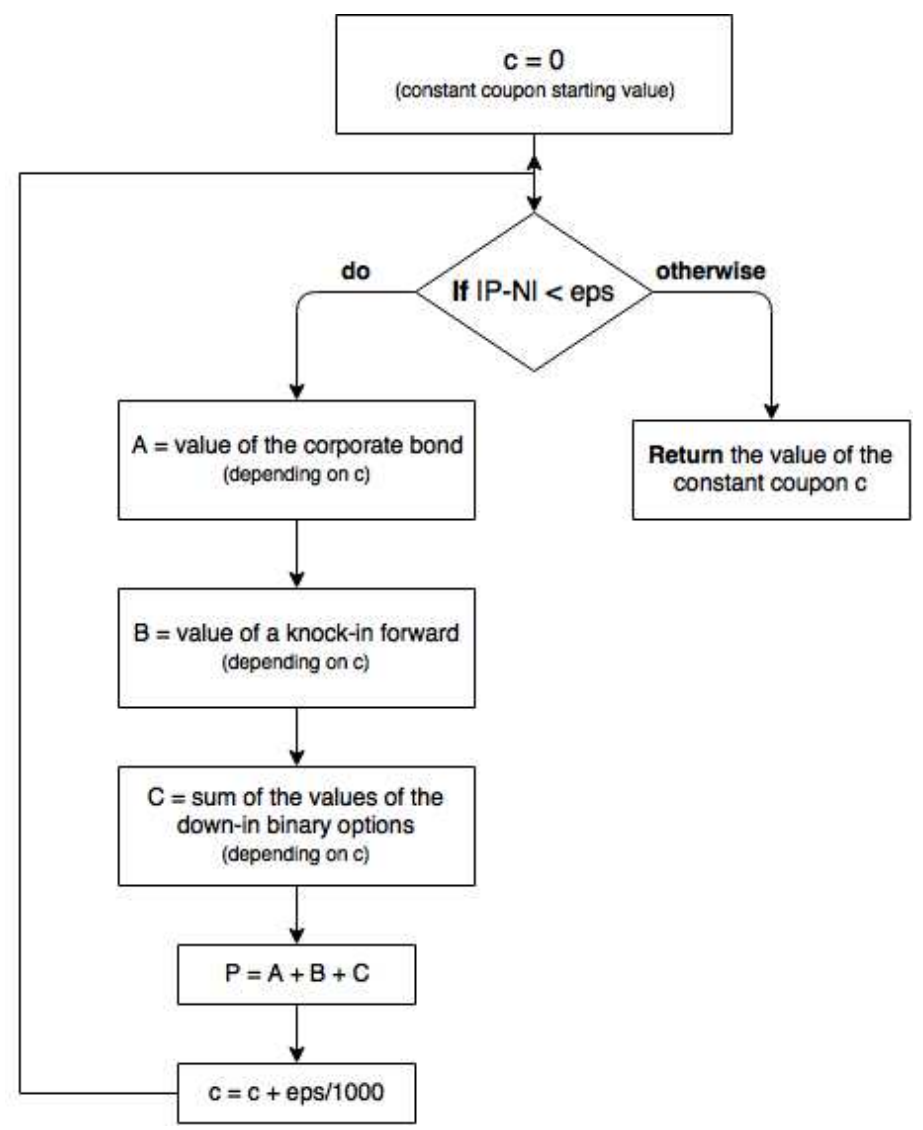

Figure 1

underlying with unknown trigger price, but with given value for the coupon offered by the CoCo contract. The parameter values are the same as the ones adopted by the most influential financial group of the United Kingdom, acting in the banking as well as in the insurance sector. The related computational evaluation is provided following the algorithm 3 in Sec. (5), which allows to determine the implicit price of the underlying trigger. In Fig. (2) we summarize the main steps of the numerical procedure by a flowchart.

Eventually, see Fig. (3), we show how the implied trigger price decrease with respect to both the value of the volatility parameter and the CoCo price. The latter is due to the effect of increasing volatility which increases the probability of hitting the trigger, implicit trigger price remaining the same. Conversely, if the investor would have to pay a higher sum to hold the contract, it will be 
guaranteed to him a lower conversion probability.

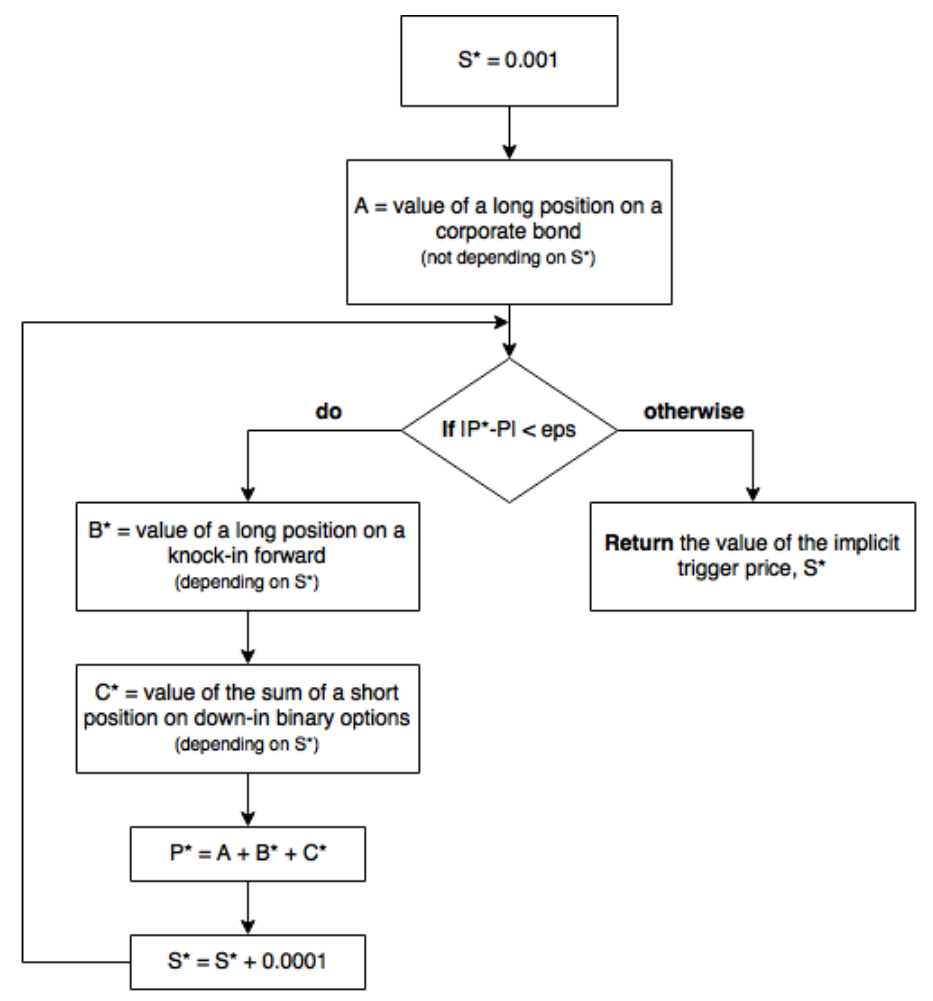

Figure 2

In what follows we will consider real CoCo contracts belonging to the portfolio under the management of a rather big italian financial company. The parameters values which are necessary to run an appropriate numerical analysis, have been estimated using the time series related to one of the leader banking group in Spain having relevant business all over the world. In particular we determine the associated CoCo implicit trigger price. To what concerns the aforementioned time series that have to be used to calibrate the model, we hvae that on 15 May 2015 the share price was $9.026 €$ and the market price of the CoCo was $102.400 €$. The contract provides a quarterly coupon of $7 \%$ of face value from May 19, 2015, and expires on Feb 19, 2019. The conversion price is determined by the greater of the stock price, the floor price $(4.50 €)$ and the par value. Other parameters needed are the volatility, the dividend yield of the title, and the continuous interest rate. We have used the implied volatility, while for the dividend yield we have taken into consideration the risk-metrics 


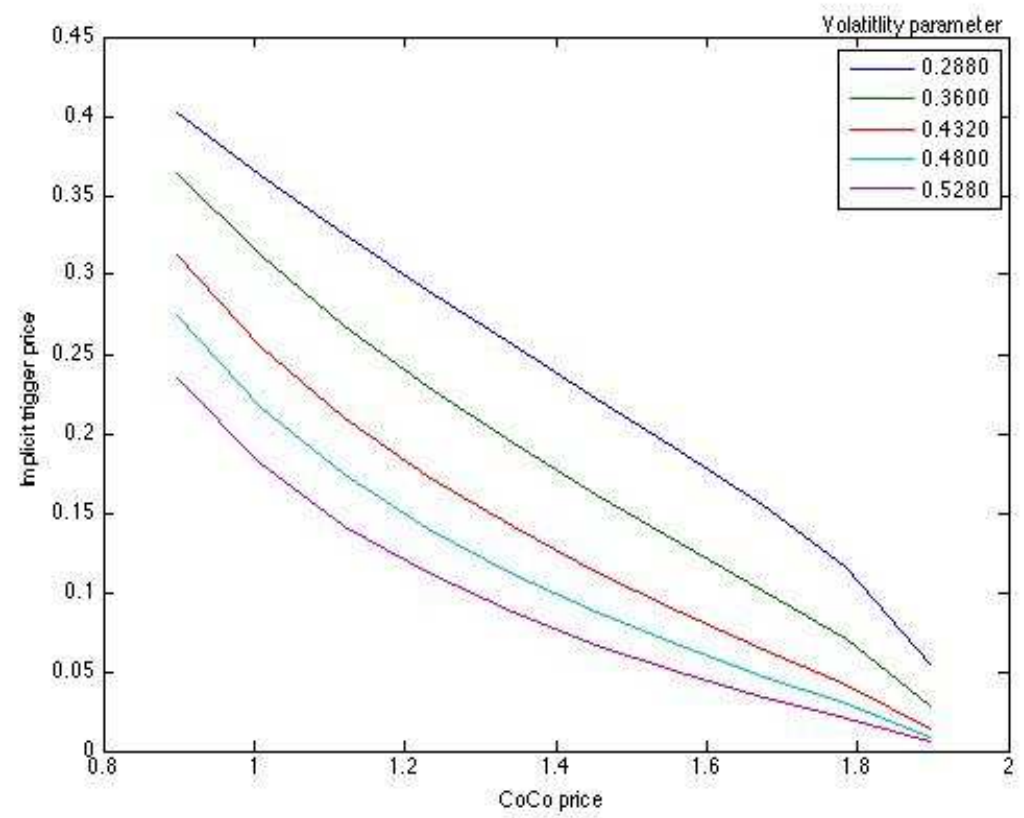

Figure 3: Implicit trigger price as a function of the CoCo contract price for different volatility values.

method, see, e.g., $[3,11,12]$, with parameter $\lambda=0.94$ on the log-returns of stock. Then we have multiplied the latter by $\sqrt{250}$, to obtain the annual value. The continuous interest rate $r$ is obtained from the risk-metrics estimation of the Euribor at 3 month, that we indicate by $e 3 m$, hence we have

$$
r=4 \log (1+e 3 m / 4) .
$$

In Sec. (5), see algorithm 4, we show how to compute the related trigger price, which turns to be equal to $7.57 €$. This value is not too far from the present value of the underlying, therefore it should not be needed an extreme collapse of the price of the underlying, but rather a moderate depreciation.

\section{Conclusions}

In the present paper we have shown how to compute the implicit trigger price of the underlying by using real market data. Such a value is highly significant since 
the underlying price is continuously available, hence it allows to precisely and continuously monitore the distance from the conversion event related to a CoCo bond type contract. It is worth to mention that usually banks tend to avoid using a trigger linked to the market value of the underlying. In fact in such a case, the conversion may be caused by market manipulation. For example, the latter is the case when the underlying price is already very close to the trigger price, so that the selling of a large quantity of actions by a securities holder could induce the conversion. A trigger priced defined as the average value of the underlying price can be used as a valid solution to the above problem.

\section{Algorithms}

\section{Algorithm 1}

The following algorithm automates the yield derivation, using a Credit Derivative approach, for particular values of the involved parameters.
1: $q \leftarrow 0$
2: $r \leftarrow 0.04$
$\triangleright$ continuous dividend yield
3: $\sigma \leftarrow 0.3$ $\triangleright$ continuous interest rate
4: $T \leftarrow 10$ $\triangleright$ maturity of the CoCo
5: $S \leftarrow 100$
6: $S_{t} \leftarrow S / 2$
7: $C_{p} \leftarrow S$
8: $\mu \leftarrow r-q-\sigma^{2} / 2$
9: $\quad N_{1} \leftarrow \Phi\left(\frac{\log \left(S_{t} / S\right)-\mu T}{\sigma \sqrt{T}}\right)$
10: $N_{2} \leftarrow \Phi\left(\frac{\log \left(S_{t} / S\right)+\mu T}{\sigma \sqrt{T}}\right)$
11: $p^{*} \leftarrow N_{1}+\left(\frac{S_{t}}{S}\right)^{2 \mu / \sigma^{2}} N_{2} \triangleright$ probability that the trigger is going to take place
12: $\lambda_{\text {Trigger }} \leftarrow-\frac{\log \left(1-p^{*}\right)}{T}$
13: $R_{C o C o} \leftarrow S_{t} / C_{p}$
14: $c s_{C o C o} \leftarrow\left(1-R_{C o C o}\right) \lambda_{\text {Trigger }}$
$\triangleright$ trigger intensity
15: $y \leftarrow r+\operatorname{cs}_{\mathrm{CoCo}}$
$\triangleright$ recovery rate of the triggering
$\triangleright$ credit spread
$\triangleright$ total continuous yield
Where $\Phi$ indicates the cumulative distribution function of the Gaussian 1- dimensional random variable, hence we get that the yield turns out to be $y=0.0730$, with $p^{*}=0.4830, \lambda_{\text {Trigger }}=0.0660, c s_{C o C o}=0.0330$. 


\section{Algorithm 2}

1: $T \leftarrow 5$

2: $N \leftarrow 1000$

3: $S \leftarrow 100$

4: $\sigma \leftarrow 0.45$

5: $r \leftarrow 0.03$

6: $q \leftarrow 0$

7: $S^{*} \leftarrow S / 4$

8: $C_{p} \leftarrow S$

9: $C_{r} \leftarrow N / C_{p}$ $\triangleright$ maturity of the $\mathrm{CoCo}$ $\triangleright$ face value del contingent convertible (in 5 years) $\triangleright$ current share price $\triangleright$ volatility $\triangleright$ continuous interest rate $\triangleright$ continuous dividend yield $\triangleright$ trigger share price $\triangle$ conversion price $\triangleright$ conversion rate

We aim at determining the value of the constant coupon, so that the CoCo value equals the face value, i.e. the par price, hence $P=N$, therefore follows that the value of the coupon c can be evaluated as follows:

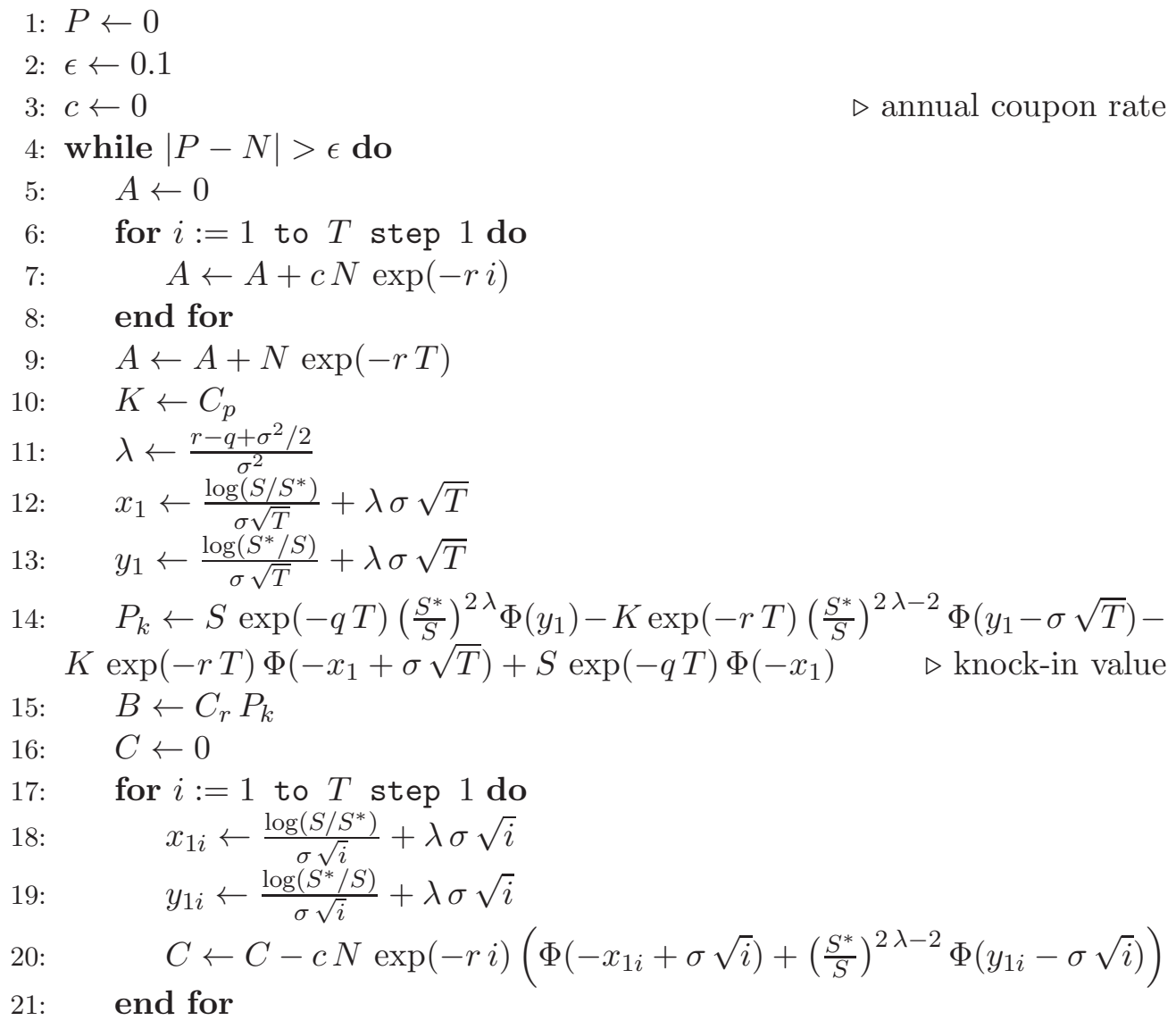




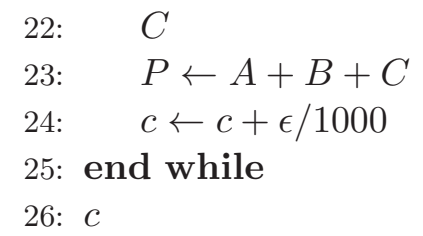

$\triangleright$ annual coupon rate

The resulting annual constant coupon is equal to $c=7.62 \%$.

\section{Algorithm 3}

Let us begin fixing some characteristic values for the involved parameters

1: $P \leftarrow 1.3976$

2: $r \leftarrow 0.0334$

3: $q \leftarrow 0.015$

4: $\sigma \leftarrow 0.48$

5: $S \leftarrow 0.47$

6: $C_{p} \leftarrow 0.59$

7: $C_{r} \leftarrow 1.695$

8: $c \leftarrow 0.15 / 2$

9: $t_{1} \leftarrow$ date $(21 / 07 / 2011)$

10: $t_{0} \leftarrow$ date $(10 / 06 / 2011)$

11: $T \leftarrow$ date $(21 / 12 / 2019)$ $\triangleright$ price of the CoCo $\triangleright$ continuous interest rate $\triangleright$ continuous dividend yield $\triangleright$ volatility $\triangleright$ current underlying share price $\triangleright$ conversion price $\triangleright$ conversion rate $\triangleright$ coupon rate $\triangleright$ first coupon payment date $\triangleright$ pricing date $\triangleright$ expiry

In what follows we consider the CoCo bond as the sum of the three components introduced in Sec. (4), namely $P=A+B+C$ where

A is a long position on a corporate bond;

$\mathrm{B}$ is a long position on a knock-in forward;

$\mathrm{C}$ is the sum of the short positions on down-and-in binary options which offsets the coupons in the case the trigger event occurs;

then we create a vector containing the coupons payment dates:

1: $i \leftarrow 2$

2: $t(1) \leftarrow t_{1}$

3: $t s(1) \leftarrow\left|t(1)-t_{0}\right| / 365$

4: while addtodate $(t(i-1), 6$ months $)<T$ do

5: $\quad t(i) \leftarrow$ addtodate $(t(i-1), 6$ months $)$

6: $\quad t s(i) \leftarrow\left|t(i)-t_{0}\right| / 365 \quad \triangleright$ time step interval between $t_{0}$ and each coupon payment (in years)

7: $\quad i \leftarrow i+1$ 


\section{8: end while \\ 9: $n \leftarrow i$ \\ 10: $\operatorname{ts}(n) \leftarrow\left|T-t_{0}\right| / 365$}

Therefore, see also the flowchart in Fig. (2), we can determine the value of the underlying implicit trigger price as follows

1: $S_{i m p}^{*} \leftarrow 0.001$

2: $P_{i} \leftarrow 0$

$\triangleright$ implicit trigger share price starting value

3: $\epsilon \leftarrow 0.001$

4: $A \leftarrow 0$

5: for $i:=1$ to $n$ step 1 do

6: $\quad A \leftarrow A+c \exp (-r t s(i))$

7: end for

8: $A \leftarrow A+\exp (-r t s(n))$

9: while $\left|P_{i}-P\right|>\epsilon$ do

10: $\quad \bar{S} \leftarrow S_{i m p}^{*} / S$

11: $\quad K \leftarrow C_{p}$

12: $\quad \lambda \leftarrow \frac{r-q+\left(\sigma^{2}\right) / 2}{\sigma^{2}}$

13: $\quad x_{1} \leftarrow-\frac{\log (\bar{S})}{\sigma \sqrt{t s(n)}}+\lambda \sigma \sqrt{t s(n)}$

14: $\quad y_{1} \leftarrow \frac{\log (\bar{S})}{\sigma \sqrt{t s(n)}}+\lambda \sigma \sqrt{t s(n)}$

15: $\quad P_{k} \leftarrow S \exp (-q t s(n))(\bar{S})^{2 \lambda} \Phi\left(y_{1}\right)-K \exp (-r t s(n))(\bar{S})^{2 \lambda-2} \Phi\left(y_{1}-\right.$ $\sigma \sqrt{t s(n)})-K \exp (-r t s(n)) \Phi\left(-x_{1}+\sigma \sqrt{t s(n)}\right)+S \exp (-q t s(n)) \Phi\left(-x_{1}\right)$ $\triangleright$ knock-in value

16: $\quad B_{i} \leftarrow C_{r} P_{k}$

17: $\quad C_{i} \leftarrow 0$

18: $\quad$ for $i:=1$ to $n$ step 1 do

19: $\quad x_{1 i} \leftarrow-\frac{\log (\bar{S})}{\sigma \sqrt{t s(i)}}+\lambda \sigma \sqrt{t s(i)}$

20: $\quad y_{1 i} \leftarrow \frac{\log (\bar{S})}{\sigma \sqrt{t s(i)}}+\lambda \sigma \sqrt{t s(i)}$

21: $\quad C_{i} \leftarrow C_{i}-c \exp (-r t s(i))\left(\Phi\left(-x_{1 i}+\sigma \sqrt{t s(i)}\right)+(\bar{S})^{2 \lambda-2} \Phi\left(y_{1 i}-\right.\right.$ $\sigma \sqrt{t s(i)}))$

22: $\quad$ end for

23: $\quad P_{i} \leftarrow A+B_{i}+C_{i}$

24: $\quad S_{i m p}^{*} \leftarrow S_{i m p}^{*}+0.0001$

25: end while

26: $S_{i m p}^{*}$

27: $A$

28: $B_{i}$ 
29: $C_{i}$

Eventually, we obtain that the implicit trigger price is $S_{\text {imp }}^{*}=0.0994$, and we get the following values for components $A=1.923, B_{i m p}=-0.288$ and $C_{i m p}=-0.238$.

\section{Algorithm 4}

Let us first set the values for the parameters characterizing our financial framework

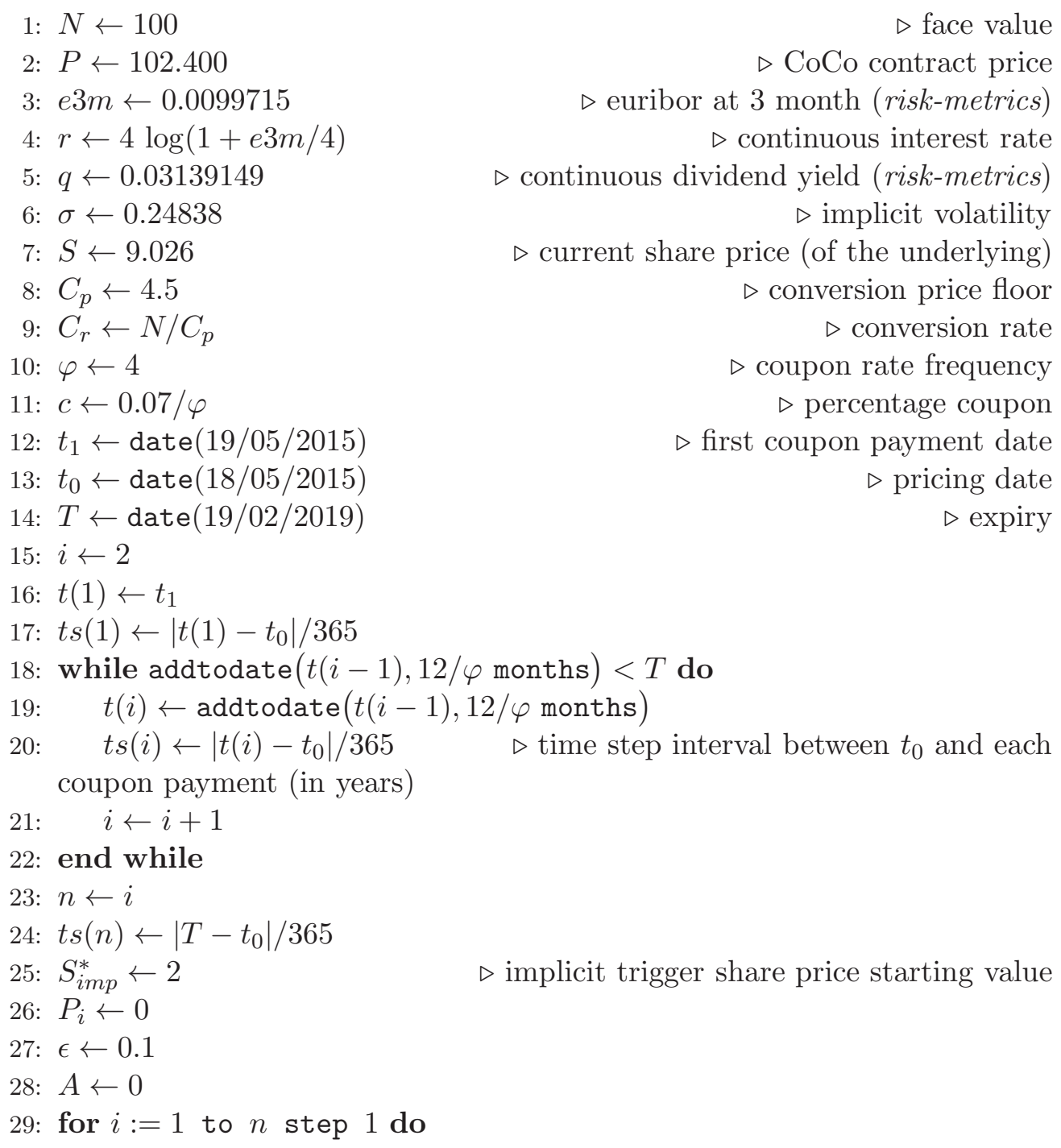




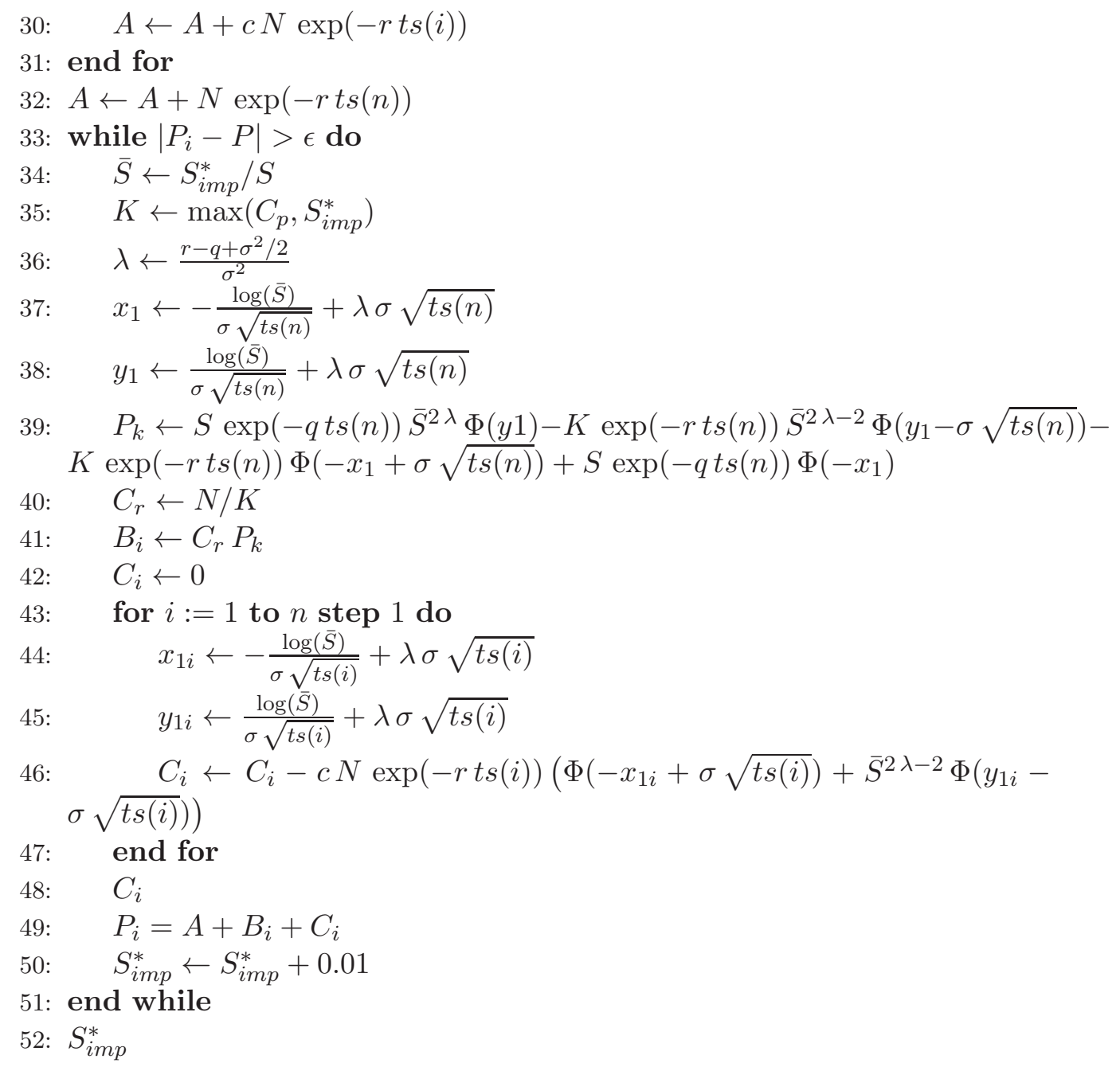

Notice that $K$ in the while loop is no longer equal to $C_{p}$, instead it equals the highest value between the conversion price and the implicit trigger price. The resulting implicit trigger price is $7.57 €$, which is a value not too far from the underlying present value. The latter result implies that it should not be needed an extreme collapse of the price of the underlying, but rather a moderate depreciation. 


\section{Glossary}

Accounting Trigger If the value of the core tier 1 approaches the null value, then the value of the subordinated bond is reset. In these cases the CoCo value will be determined by a basic trinomial model. With probability $p_{1}$ the CoCo assumes a null future value, since it will be converted to zero, with probability $p_{2}$ it will be converted to a value lower than the face value, and with probability $\left(1-p_{1}-p_{2}\right)$ the trigger will not be triggered and therefore there will be no conversion.

Binary option This type of option is characterized by a payoff that is limited to a fixed amount or to zero.

Board of directors This is the collegial body which is responsible for the management of a certain company.

CDS (acronym for Credit Default Swap) is a swap, hence a derivative instrument which allows for the exchange of cash flows between two counterparts. A CDS has the function of transferring the credit risk. It is classified as a hedging instrument and it is widely used in the financial arena.

Corporate Bond Corporate bonds are bonds that may be provided by banks, companies or private companies, in order to finance themselves, hence to obtain greater liquidity availability.

Credit spread This term is used to describe a series of measures which serve to determine how much an investor is paid as compensation of assuming the credit risk inherent in the title.

Dilution Diluition indicates the situation that results in a reduction of any index per share due to an increase in outstanding shares, both as a result of a new issue, whether as a result of the free distribution or conversion of bonds. The income distributed by the company will be split among several actions. Therefore, for the same total profit, earnings per share will decrease.This situation is perceived in the immediate, while in the short to medium term will be reabsorbed.

Knock-in option are derivatives belonging to the barrier options category. They are characterized by the fact that the faculty, of purchase or sale, comes into existence only when the price of the Underlying touches the predetermined barrier (knock-in level). 
Down-and-in binary option This is a particular type of knock-in binary option that becomes activated when the price of the underlying exceeds downward the level of the barrier.

Equity securities Such type of equities refer to a share of (equity) interest in an entity, such as the capital stock of a company, partnership or trust. They do not require any payment and entitle the holder to some control of the company on a pro-rata basis. The latter means that equity holders maintain voting rights and, thus, some control of the business. In the case of bankruptcy, they share only in residual interest after all obligations have been paid out to creditors. As an example, let us consider the case in which CoCo bonds are converted. Let $n^{*}$ be the number of shares pre-conversion, $n$ the number of CoCo bonds, and $n_{c}=C_{r} n$ the number of shares converted by CoCo bonds after the trigger event. Then the number of shares after conversion will be $n_{T}=n^{*}+n_{c}$, and therefore also the share price will change. Let $S^{*}$ be the share price and $N^{*}=n^{*} S^{*}$ the sum of the share face values pre-conversion, and $N_{T}=n_{T} S_{c}$ be the sum of the share face values after conversion. Since the face value doesn't change, $N_{T}=N^{*}$, the new share price will be $S_{c}=\frac{n^{*}}{n^{*}+n_{c}} S^{*}$, with clearly $\frac{n^{*}}{n^{*}+n_{c}}<1$.

Recovery rate This parameter measures the portion of the face value of an obligation that an investor expects to recover if default or devaluation events occur.

Seniority It refers to the order of repayment in the event of issuer bankruptcy or sellout.

\section{References}

[1] B. Albul, D. Jaffee, A. Tchistyi, Contingent Convertible Bonds and Capital Structure Decisions, Haas School of Business, University of California at Berkeley (2013).

[2] P. Alvemar, P. Ericson, Modelling and Pricing Contingent Convertibles, School of Business, University of Gothenburg (2012).

[3] J. Berkowitz, J. O'Brien, How Accurate Are Value-at-Risk Models at Commercial Banks ?, Journal of Finance, $\mathbf{5 7}$ (2002).

[4] D. Brigo, J. Gatcia, N. Pede, CoCo Bonds Valuation with Equity- and Credit-Calibrated First Passage Structural Models, Imperial College, London, U.K. (2003).

[5] J. De Spiegeleer, W. Schoutens, Pricing Contingent Convertibles: A Derivatives Approach, Department of Mathematics Katholieke Universiteit Leuven (2011). 
[6] European Parliament, Council of the European Union, Regulation (EU) No 575/2013 of the European Parliament and of the Council of 26 June 2013 on prudential requirements for credit institutions and investment firms and amending Regulation (EU) No 648/2012, (2013).

[7] F. Di Girolamo, F. Campolongo, J. De Spiegeleer, W. Schoutens, Contingent Conversion Convertible Bond: New avenue to raise bank capital, Department of Mathematics, Leuven, Belgium (2012).

[8] J.C. Hull, Option, futures, and other derivatives, School of management University of Toronto (2009).

[9] Lujing Su, M.O. Rieger, How Likely is it to Hit a Barrier? Thoretical and Emperical Estimates (2009).

[10] G. Pennacchi, A Structural Model of Contingent Bank Capital, College of Business, University of Illinois (2011).

[11] P. Zangari, RiskMetrics Technical Document, (1996).

[12] G. Zumbach, A gentle introduction to the RM 2006 methodology, RiskMetrics Working Paper, (2006). 
